\title{
Study of Visual Spatial Perception Performance at Close Range in Underground Coal Mine
}

\author{
Ren Dawei ${ }^{1}$, Liu Yang ${ }^{2}$ and Chen Litao ${ }^{3}$ \\ ${ }^{1}$ Department of Industrial Engineering, College of Mining and Safety Engineering \\ Shandong University of Science and Technology, Tsingtao, China \\ ${ }^{2}$ Department of Industrial Engineering, College of Mining and Safety Engineering \\ Shandong University of Science and Technology, Tsingtao, China \\ ${ }^{3}$ Department of Industrial Engineering, College of Mining and Safety Engineering \\ Shandong University of Science and Technology, Tsingtao, China \\ 19660562@qq.com,_fanxipingly@163.com
}

\begin{abstract}
In coal mines the environment is complex, crowded with personnel and equipment, as well as lack of colors. The safety in production is influenced by visual spatial perception is whether or not accurate. The visual spatial perception precision plays a significant even crucial role in positioning. By simulating the environment in coal mines, the positioning experiment about participants' visual spatial perception performance of different target objects in two models-from far to near and from near to far has been carried out. Experimental results showed that visual spatial perception distance was significantly shorter than target range, that visual spatial perception in deep pink was the poorest. In addition, the $H$ has become appreciable and regular with visual spatial perception changing. The experimental results provided references for setting warning marks, safe distances and choosing colors for infrastructure in coal mines.
\end{abstract}

Keywords: in coal mines; visual spatial perception; simulations; HIS; frequency of participants' approval

\section{Introduction}

Personnel who work in coal mines should accurately judge the relative positions within the range in the complex and crowded environment. However, they are predisposed to produce visual fatigue because of no natural light except miner's lamp. The colors in coal mines are given priority to grey and dark. Therefore, it is necessary to make a research on people's visual spatial perception performance related to colors and relative motion. Personnel who work in coal mines should accurately judge the relative positions within the range in the complex and crowded environment. However, they are predisposed to produce visual fatigue because of no natural light except miner's lamp. The colors in coal mines are given priority to grey and dark. Therefore, it is necessary to make a research on people's visual spatial perception performance related to colors and relative motion.

Visual spatial perception is the individual reflections of the concave and convex in the same object or different objects varying distances. Generally speaking, it relies on lots of objective conditions, individual's internal conditions or clues and comprehensive utilization in existing visual experiences. In summary, the clues include monocular clues and binocular ones. The former focuses on the characteristics of visual stimuli themselves, mainly including the relative sizes, shelters, texture gradient, light and shadow, perspective lines, aerial perspective and motion parallax. The later focuses on images preprocessing in the spatial frequency domain after received by two eyes. The clues processed by each channel are sent to the higher level integrated and finally 
perceived in the form of spatial perception [1].

At present, researches on factors which influence people' spatial perception performance in the close view in coal mines are significantly few, and mainly in traffic field.

Guo Weili [2] et. al., have measured spatial perception precision for 248 male truck drivers at low and high speed by using the visual space tester in the study on truck driver's visual spatial perception. Results have shown that there are no differences between truck drivers' visual spatial perception precision in safety and accident groups at low and high speed while at high speed, significant differences appeared. Working age has great influences on perception precision.

Chu Yuede [3] et. al., have revealed that basketball athletes on different levels have different depth perception in the preliminary study on. In the age group of 1-22, age does not affect depth perception at all.

Yu Hao [4] et. al., have pointed out that depth perception provides efficient references for selecting seafarers in the study on seafarers' depth perception. The depth perception is not affected by ages, working ages, and the length of service. The seafarers' depth perception is similar although they are in different positions and have different educational levels and come from different places. It is appropriate to select seafarers in terms of depth perception to some extent.

Jochen Musseler [5] et. al., have put forward that visual action is essential for a dynamic environment, especially in visual spatial perception in the study on visual spatial perception and action. It has been emphasized that they would be more efficient if they were used from different theoretical and empirical views.

Philbeck and Loomis [6] have inspected subjects' distance judgment task within the range from $79 \mathrm{~cm}$ to $500 \mathrm{~cm}$ in dark and light. There was significant consistency between oral presentation and matching blind walk in dark, namely, overestimate short distances and underestimate long ones.

Gibson, Purdy and Bergman [7] have measured subjects' absolute and relative distance judgment in the natural field. Studies have shown that participants which were not trained in advance by special exercises often had a tendency to underestimate absolute distances that were less than physical distances. In the relative distance judgment task, there was little influence on judgment precision although the two stimuli presented to the subjects on either side were different in size and shape.

Whitmer, B. G. and Sadowski, W. J. J. [8] have researched that virtual environment made distance perception distorted in a limited way .Thompson [9] et. al., believed that spatial properties were unlike between in the real and virtual environment. Studies have found that distance perception was compressed in the depth direction in the virtual environment. Ellard, C. G. and Wagar, L. S. [10] have pointed out that perceptual-motor coupling in virtual environment might be different in the real environment.

HIS (respectively Hue, Intensity, Saturation) are indexes which are commonly used to describe the color characteristics. In the visual performance research, HIS are often used as quantitative indexes for the conversion of RGB. The conversion between IHS and RGB is based on the triangle transform formula as shown in formula 1 and formula 2.

$I=\frac{1}{3}(R+G+B)$

$\left\{\begin{array}{l}B=\text { Min }, H=\frac{G-B}{3(I-B)}, S=1-\frac{B}{I} \\ R=M \text { in }, H=\frac{B-R}{3(I-R)}, S=1-\frac{R}{I} \\ G=M \text { in }, H=\frac{R-G}{3(I-G)}, S=1-\frac{G}{I}\end{array}\right.$ 
Wang Xiaoyan [11] et. al., have raised that the combination algorithm on remote sensing image of HIS based on structure similarity and wavelet transform can raise the algorithm in the fusion image's resolution ratio, and it can also maintain the spectral characteristics of original multispectral at the same time,. It is an ideal fusion method of remote sensing images.

Chai Yanmei [12] et. al., have found that a new method based on the fusion of direction information measure and IHS transform image is much more efficient than traditional IHS fusion method and IHS fusion method based on histogram matching in keeping the TM spectral information and resisting noises.

The term of "virtual reality" was initially proposed by Jaron Lanier and now is a fundamental technology in different areas, such as the automotive industry, architecture, medical training and education [13-14]. As target environments grow in size and complexity, advanced and new VR systems are emerging, such as large scale virtual manufacturing environment [15], integrated aerial virtual environment [16], hybrid display system [17] and other types of VR systems.

Related researches have yet proved that some spatial knowledge in the virtual environment can be transformed to the real environment, such as the judgment on path distance, determination of the location of the objects[18-19]. And it also proved that virtual reality technology in spatial cognition was effective [20]. Information platform for improving the ability was developed consistent with realistic needs [21].

\section{Experimental Methods}

Based on existing researches, a certain number of people were chose as participants in the positioning experiment to measure the cognitive distances of different target objects by simulating the visual environment in coal mines. It was simulated environment in coal mines as shown in Figure 1. It includes a simple closed opaque simulated structure and an Installed door between the roadways or roadway ports on the surface. When the door is closed, the whole roadway will be in a completely sealed and opaque state. At the top of its profile, door and the floor are made of opaque material and black emulsion varnish. There are a real smoke generator and collector, a leather measuring tape, a white lab bench, a simulated roadway, an air conditioning, some colorful paper cubic target objects, a coal dust generator and collector. Figure 2 shows the appearance of the simulated environment.

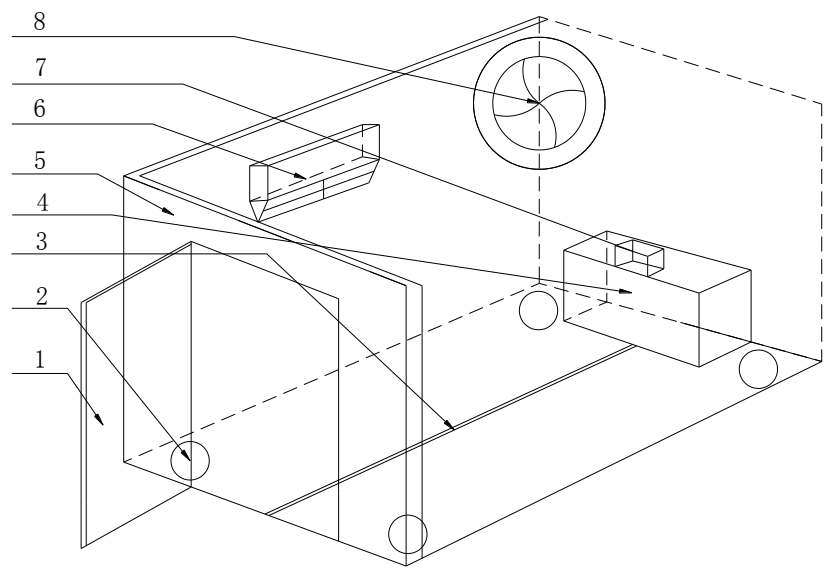

Figure 1. Simulated Environment in Coal Mines 
1. Installed door between the roadway or roadway port

2. Real smoke generator and collector

3. Leather measuring tape

4. White lab bench

5. Simulated roadway

6. Air conditioning

7. A colorful paper cubic target object

8. Coal dust generator and collector

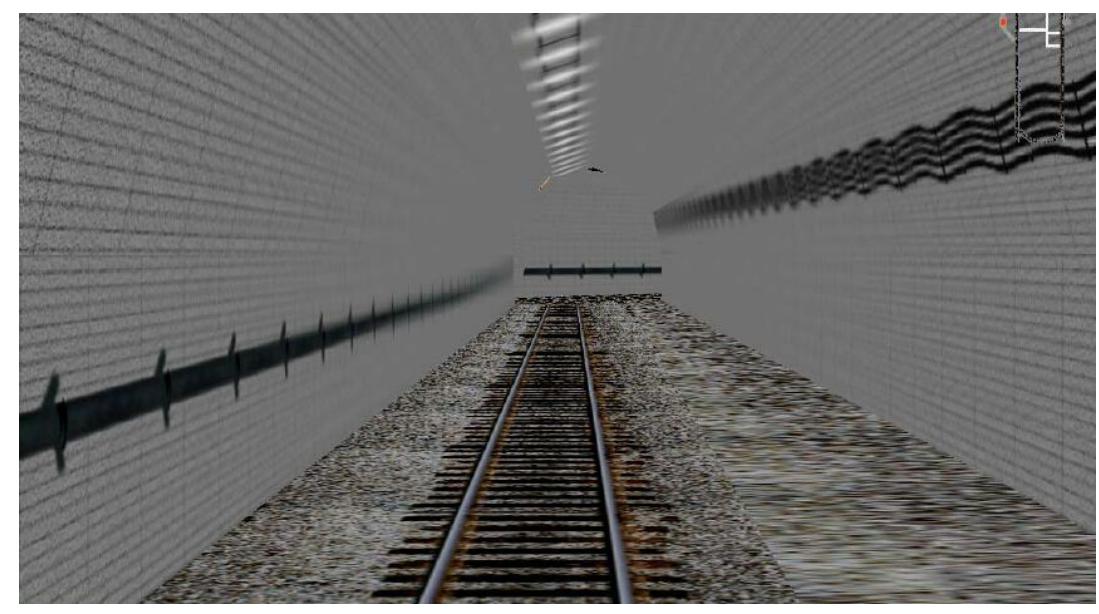

\section{Figure 2. The Appearance of the Simulated Environment}

The rest experimental materials included some colorful paper cubic target objects which are in the same size $(18 \mathrm{~cm} \times 18 \mathrm{~cm} \times 18 \mathrm{~cm})$ and shape, a leather measuring tape, a simulated adjustable miner's lamp, an illuminometer, a luminometer, a hygrothermograph, lab records and other supporting tools. The RGB and HIS about selected objects' color are listed in Table 1.

\subsection{Simulated Environment in Coal Mines}

The simulated environment in coal mines primarily referred to the visual environment. In the experiment, a rectangular space whose length, width and height were respectively $500 \mathrm{~cm}, 220 \mathrm{~cm}$ and $270 \mathrm{~cm}$ was built by using blocking materials. All it was covered with a black cloth. There was an exit for participants' getting in and out and moving, and on the other end, target objects were placed on a $120 \mathrm{~cm}$-high black bench. The reflective rate of the black cloth is about 3.5\% [22]. Equipment and devices were installed in the simulated roadway for implementation, just like moisture and humidity control equipment, microphone and sound equipment, as well as coal dust generator and collector, real smoke generator and collector. 
Table 1. Transform between RGB and HIS

\begin{tabular}{|c|c|c|c|c|c|c|}
\hline colors & $\mathrm{R}$ & G & B & I & $\mathrm{H}$ & $\mathrm{S}$ \\
\hline Deep pink & $5^{25}$ & 20 & $7^{14}$ & $1^{14}$ & 0.65 & $6^{0.8}$ \\
\hline pink A & $5^{25}$ & 105 & $0^{18}$ & $0^{18}$ & 0.62 & $2^{0.4}$ \\
\hline pink B & $5^{25}$ & 192 & $3^{20}$ & $7^{21}$ & 0.85 & $1^{0.1}$ \\
\hline $\begin{array}{l}\text { Orchid } \\
\text { purple }\end{array}$ & $8^{21}$ & 112 & $4^{21}$ & $1^{18}$ & 0.51 & $8^{0.3}$ \\
\hline sky blue & 0 & 191 & $5^{25}$ & $9^{14}$ & 0.57 & $0^{1.0}$ \\
\hline blue green & 0 & 255 & $7^{12}$ & $7^{12}$ & 0.33 & $0^{1.0}$ \\
\hline forest green & 34 & 139 & 34 & 69 & 1.00 & $1^{0.5}$ \\
\hline gray & $8^{12}$ & 128 & $8^{12}$ & $8^{12}$ & 0 & 0 \\
\hline yellow & $5^{25}$ & 255 & 0 & $0^{17}$ & 0.50 & $0^{1.0}$ \\
\hline orange & $5^{25}$ & 165 & 0 & $0^{14}$ & 0.39 & $0^{1.0}$ \\
\hline
\end{tabular}

\subsection{Participants}

A total of 20 male participants between the ages of 18 and 23 (mean $(M)=21.45$ years, standard deviation $(\mathrm{SD})=28.95$ years) were recruited from Shandong University of Science and Technology. All participants' corrected vision was above 1.0 and there was no color blindness and color amblyopia. Their cognitive levels are approximately same and they have not taken part in similar experiments before.

\subsection{Processes}

Step 1: The participants were required to get together in the laboratory at 8 p.m. All windows were covered by curtains to prevent outside light coming in. Then, turn on all lamps and place the black cubic target object on the $120 \mathrm{~cm}$-high white bench. Four observation points were set respectively in 4 places $-300 \mathrm{~cm}, 350 \mathrm{~cm}, 400 \mathrm{~cm}$ and $450 \mathrm{~cm}$ away from the target object which are called target ranges. Let participants stand on every point in turn to watch the target object for about 15 minutes. In the experiment, some environmental indexes were as follows: the temperature in simulated environment was $25^{\circ} \mathrm{C}$; the relative humidity was $60 \%$; the average illumination in indoor was $150.31 \mathrm{x}$; the average illumination in simulated environment in coal mines was $0.51 \mathrm{x}$ and the illumination of simulated miner's lamp was $1501 x$.

Step 2: The participants were allowed into the simulated environment in coal mines in turn. With the help of miner's hand lamp, all were required to watch the pink-A target object and then position the above four different observation points from near to far in 8 seconds. The experimenter recorded the different locations that participants have judged respectively. The distance between target object and a participant was called "visual spatial perception distance".

Step 3: Change the target object's color into pink-B, deep red, orchid deep purple, sky blue, blue green, forest green, gray, yellow, orange target object in turn and repeat step 2 for 9 times. 
Step 4: This time, participants were required to watch the target object and then position the above four different observation points from far to near. The target object's color was respectively changed into deep red, turquoise, forest green, pink-A, gray. Repeat step 2 for 5 times.

\section{Analysis and Discussion}

In this experiment, during the observation in various object's color from near to far, 400 records on visual spatial perception were obtained, and during the observation in various object's color from far to near, 200 records were acquired. The participants' visual spatial perception distances in the forest green are shown in Table 2.

Table 2. Visual Spatial Perception Distance about Forest Green Object

\begin{tabular}{ccccccccccc}
\hline Ranges & P1 & P2 & P3 & P4 & P5 & P6 & P7 & P8 & P9 & P10 \\
\hline 300 & 414 & 364 & 347 & 326 & 320 & 337 & 339 & 325 & 351 & 360 \\
350 & 454 & 414 & 397 & 386 & 391 & 398 & 409 & 390 & 412 & 414 \\
400 & 502 & 459 & 464 & 464 & 457 & 455 & 465 & 450 & 464 & 464 \\
450 & 558 & 505 & 521 & 520 & 518 & 509 & 515 & 507 & 513 & 511 \\
\hline
\end{tabular}

\subsection{Analysis}

The analysis was mainly about two sides: first, the relationship between the average participants' visual spatial perception distance and the target range; secondly, the relationship between the IHS characteristics of target objects in different colors and visual spatial perception.

3.1.1. Relationship between the Average Distance and the Target Range: Table 3 is a comparison table about the average participants' visual spatial perception distance, the average visual spatial perception distance from far to near and from near to far and the target range. The average was bigger than $t$ target range, and there was the linear relationship between them in regression analysis. The coefficient of determination of goodness of fit was bigger than $99.99 \%$. Their fitting equations are shown in formula 3 , formula 4 and formula 5 .

Table 3. Average Participants' Visual Spatial Perception Distance and Target Range

\begin{tabular}{cccc}
\hline range & $Y_{\text {far } \rightarrow \text { near }}$ & $Y_{\text {near } \rightarrow \text { far }}$ \\
$(X)$ & $Y_{\text {all }}$ & 329.75 & 311.8 \\
\hline 300 & 318.53 & 382.3 & 361.9 \\
350 & 369.55 & 436.67 & 413.59 \\
400 & 422.24 & 489.93 & 464.33 \\
450 & 473.93 & & \\
\hline
\end{tabular}

$Y_{a l 1}=1.0378 X+6.8937$ 


$$
\begin{aligned}
& Y_{\text {far } \rightarrow \text { near }}=1.0698 X+8.48 \\
& Y_{\text {near } \rightarrow \text { far }}=1.01086 X+5.945
\end{aligned}
$$

About $99.99 \%$ records can be covered by the above fitting equations. The T-Test $(\alpha=0.05)$ have shown that participants' visual spatial perception distance were quite different from the target range. And by using F-Test $(\alpha=0.05)$, there was significant differences between visual spatial perception distance from far to near and from near to far.

3.1.2. Relationship between IHS Characteristics and Visual Spatial Perception Distance: When the target range remained the same, the change tendency between frequency of participants' approval of visual spatial perception and $\mathrm{H}, \mathrm{S}$ was shown in Figure 3. The fitting equations of the four lines were as formula 6 , formula 7 , formula 8 and formula 9 .

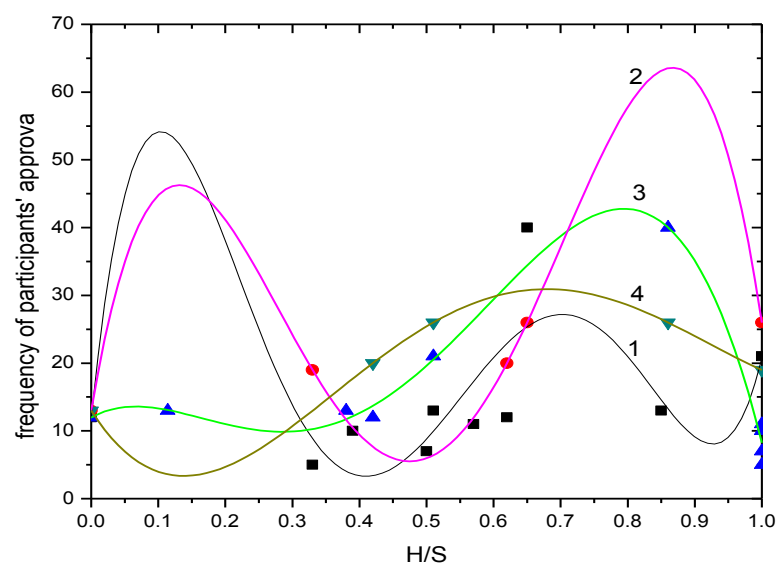

Figure 3. The Tendency Chart between Frequency of Participants' Approval and $\mathrm{H}, \mathrm{S}$

$$
\begin{aligned}
& y_{1}=6998.9 x^{5}-18747 x^{4}+17850 x^{3}-7060.8 x^{2}+967.5 x+11.946 \\
& y_{2}=2683.8 x^{4}+5275.6 x^{3}-3162.6 x^{2}+583.76 x+13 \\
& y_{3}=141.16 x^{5}-1095.2 x^{4}+1435.5 x^{3}-539.34 x^{2}++54.143 x+11.975 \\
& y_{4}=375.48 x^{4}-962.29 x^{3}+748.64 x^{2}-155.83 x+13
\end{aligned}
$$

When $\mathrm{H}<0.5$, the change tendency of visual spatial perception from far to near and from near to far was almost the same with $\mathrm{H}$ increasing and the frequency of participants' approval on every color was consistent with each other. When $\mathrm{H}>0.5$, the change range from far to near was greater than that from near to far with $\mathrm{H}$ increasing. With the $\mathrm{S}$ increasing, the change tendency of visual spatial perception from far to near and from near to far was roughly in the same, and the frequency of participants' approval on every color was consistent. 


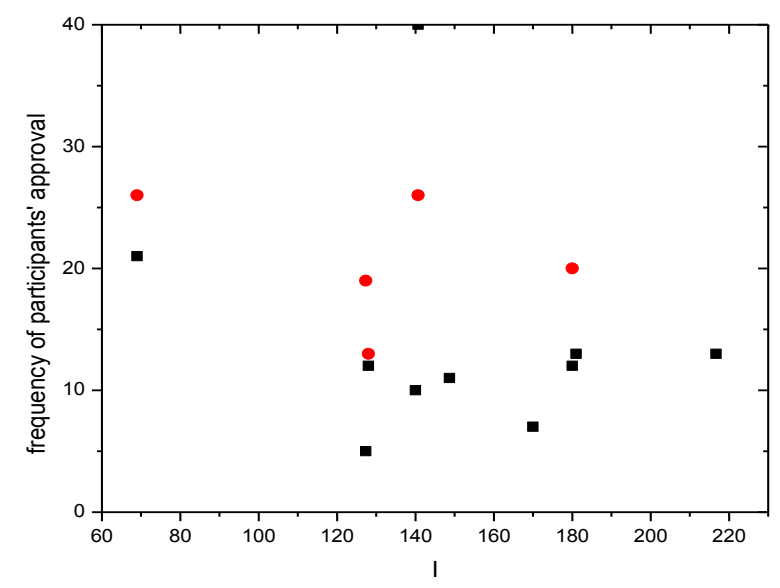

\section{Figure 4. The Tendency between the Frequency of Participants' Approval} and I

Figure 4 shows the change tendency of the frequency of participants' approval of visual spatial perception distance and I. With I increasing or decreasing, the frequency of participants' approval on all colors was unconspicuous.

\subsection{Discussion}

By the analysis of experimental data, the results are as follows:

(1) There are clear differences between participants' visual spatial perception distance and the target range. All the average participants' visual spatial perception distance is less than target range, and there is a linear relationship between them.

(2) In the experiment, the participants' visual spatial perception distance in deep red is the shortest and the frequency of participants' approval is maximum.

(3) In IHS characteristics, the changes of $\mathrm{H}$ have clear influences on participants' visual spatial perception. There are significant differences between visual spatial perception for target objects from far to near and from near to far especially when $H>0.5$; With $\mathrm{S}$ increasing, visual spatial perception for target objects from far to near and from near to far has almost the same tendency; The changes of I does not clearly affect participants' visual spatial perception.

\section{Summary}

Participants' visual spatial perception is significantly affected by simulated environment in coal mines through the experiment. Visual spatial perception in deep red is poorest, and the changes of $\mathrm{H}$ and $\mathrm{S}$ have clear influences on participants' visual spatial perception. It has researched the person's visual spatial perception in the coal mine environment in the color of the visual qualitative description on the target objects using HIS system. It helped realize safety production in coal mines with cognitive psychology, ergonomics, virtual reality platform, facilities planning, philosophy and other multidisciplinary. There are clear differences between participants' visual spatial perception distance and the target range. All the average participants' visual spatial perception distance is less than target range, and there is a linear relationship between them. In the experiment, the participants' visual spatial perception distance in deep red is the shortest and the frequency of participants' approval is maximum. It helped provide 
support for the establishment of safety distance as well as give full consideration to visual spatial perception error in the underground environment. It helped improve ideas for the underground working environment and effectively prevent accidents caused by underground environmental factors in the workplace. In IHS characteristics, the changes of $\mathrm{H}$ have clear influences on participants' visual spatial perception. There are significant differences between visual spatial perception for target objects from far to near and from near to far especially when; With S increasing, visual spatial perception for target objects from far to near and from near to far has almost the same tendency; The changes of I does not clearly affect participants' visual spatial perception. It helped logo design and optimization and provided references for setting warning marks, safe distances and choosing colors for infrastructure in coal mines. However, the shortages of the positioning experiment are that some indexes have not been considered such as coal dust, ventilation, psychological and physiological factors and so on. In addition, many factors, such as the shapes, sizes and colors of target object, the different number of experimental records in the two positioning processes, may affect the accuracy of visual spatial perception in the experiment, which needs to be further verified.

\section{Acknowledgment}

This work is partially supported by REN DAWEI. The authors also acknowledge gratefully the helpful comments and suggestions of the reviewers, which have improved the presentation.

\section{References}

[1] Yao Guozheng, J., "The information on spatial perception was processing in the monocular cues", Progress in Biochemistry and Biophysics, vol. 4, (1984).

[2] Guo Weili, J., "Study on truck driver's visual spatial perception”, Chinese Journal of Ergonomics, vol. 5, no. 3, (1999).

[3] Chu Yaode, J., "Preliminary study on perceptual ability among basketball players", Chinese Journal of Applied Psychology, vol. 4, (1988).

[4] Yu Hao, J., "Study on the depth perception for seafarers. Chinese Journal of Nautical Medicine and Hyperbaric Medicine", vol. 3, no. 1, (2006).

[5] Jochen Musseler, J., "Visual space perception and action: Introductory remarks", Visual Cognition, vol. $1,(2010)$.

[6] J. W. Philbeck and J. M. Loomis, J., "Comparison of two indicators of perceived egocentric distance under full-cue and reduced-cue conditions", Journal of Experimental Psychology: Human Perception and Performance, vol. 23, no. 1, (1999).

[7] E. J. Gibson, Bergman, J. Rand and Purdy, J., "The effect of prior training with a scale of distance on absolute and relative judgments of distance over ground", Psychol, vol. 50, (1955).

[8] B. G. Whitmer and W. J. Sadowski, J., "Nonvisually guided locomotion to a previously viewed target in real and virtual environments", Human Factors, vol. 40, no. 3, (1998).

[9] W. B. Thompson, P. Willemsen and A. A. Gooch, J., "Does the quality of the computer graphics matter when judging distances in visually immersive environments", Presence, vol. 13, (2004).

[10] C. G. Ellard and L. S. Wagar, J., "Plasticity of the association between visual space and action space in a blind-walking task", Perception, vol. 37, no. 7, (2008).

[11] Wang Xiaoyan, J., "A Kind of Based on Structure Similarity of the Combination of IHS and Wavelet Transform Algorithm Used in Remote Sensing Image", Journal of Lanzhou University (Natural Sciences), vol. 47, no. 5, (2011).

[12] Chai Yanmei. J., "A Kind of Based on Direction Information Measure and IHS Transform of a New Method of Image Fusion”, Journal of Northwestern Polytechnical University, vol. 22, no. 4, (2004).

[13] D. Waller, J., "The hive: a huge immersive virtual environment for research in spatial cognition", Behavior Research Methods, vol. 39, no. 4, (2007).

[14] E. D. Ragan, D. A. Bowman and K. J., "Huber, J. Supporting cognitive processing with spatial information presentations in virtual environments", Virtual Reality, vol. 16, no. 4, (2012). 
[15] H. Lee and A. Banerjee, J., "A self-configurable large-scale virtual manufacturing environment for collaborative designers", Virtual Reality, vol. 15, no. 1, (2011).

[16] M. Amiri Atashgah and S. M. B. Malaek, J., "An integrated virtual environment for feasibility studies and implementation of aerial monoslam", Virtual Reality, vol. 16, no. 3, (2012).

[17] S. Lee, Editors, "Design and evaluation of a hybrid display system for motion-following tasks", In: Gerhard Goos, Juris Hartmanis, and Jan van Leeuwen, eds. Proceedings Second International Conference, ICVR 2007 (part of HCI International 2007), Springer, Beijing, China, (2007).

[18] Wen Zhonglin, Hou Jietai, W. Marsh and Herbert, J., "Structural equation model test fitting index and CFI square criterion”, Acta Psychologica Sinica, vol. 36, no. 2, (2004).

[19] Lin Wenying and Hou Jietai, J., "Analysis of Correlational Data: An Introduction to Confirmatory Factor Analysis and Strutural Equation Modelling”, Educational Psychology, vol. 23, no. 1, (1995).

[20] M. Mulinge and C. W. Mueller, J., "Employee Job Satisfaction in Development Countries: The case of Kenya", World Development, vol. 26, no. 12, (1998).

[21] W. M. Hsieh, Chen CC, Wang SC, Tan SY, Hwang YS, Chen, SC, Lai, JS and Chen YL, J., "The Virtual reality system -based on device for the elderly in fall prevention", Journal of Technology Health Care, vol. 22, no. 1, (2014).

[22] Zhou Ren, J., "The Smallest Reflective Rate of Coal and Reflective Rotation Angle Test", Coal Geology and Exploration, vol. 6, (1980).

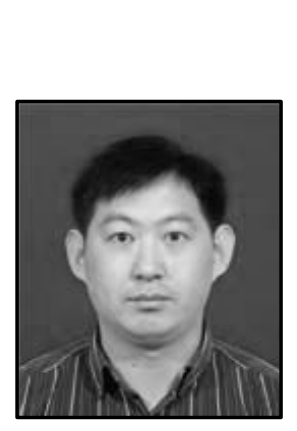

Authors

Ren Dawei, male, born in July, 1979 works in College of Mining and Safety Engineering, Shandong university of science and technology and is the deputy director for industrial engineering; $\mathrm{He}$ is mainly engaged in the teaching and researches about safe management, industrial engineering and other aspects in coal mines, and Successively presided and participated in a number of scientific researches. In recent years, He teaches mainly computer application basic principle (database), logistics, mining enterprises management courses for the undergraduate and graduate students.

Published by the nearly five years:

Ren Dawei, Yang Ning, Coal Mine Dispatching Model Research Based on PFS, 2009 International Conference on Information Management, Innovation Management and Industrial Engineering, ISBN-13: 9780769538761.

Ren Dawei, Zhao Shangwu, Process Analysis and Optimization of Hospital Out-patient Flow Based on Arena, 2009 IEEE 16th International Conference on Industrial Engineering and Engineering Management, 2009, 10, ISBN-13: 9781424436705.

Ren Dawei, Yang Ning, Monitoring Research of Mine IFH Based on IVR, 2009 International Symposium on Computational Intelligence and Design, 2009, 12, ISBN-13: 9780769538655.

Ren Dawei, Gai Lili, Study of Destruction Rule in Shaft Diameter Variation Area Based on FLAC3D, International Conference on Electrical and Control Engineering, 2012, 6, ISBN-13: 9780769540313.

Ren Dawei, Yang Ning, Optimization and design of coal mine safety supervision information flow based on internet/intranet, Applied Mechanics and Materials, 2011, 6, ISSN: 16609336; ISBN13: 9783037851371. 


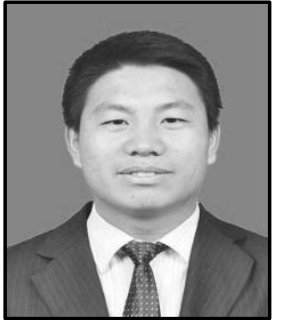

Liu Yang, male, born in March, 1991 studies in College of Mining and Safety Engineering, Shandong university of science and technology and his major is industrial engineering; He is mainly engaged in the researches about industrial engineering, human factors engineering, ergonomics, man-machine interface and other aspects in pipeline engineering, especially in coal mines. He has participated in a number of scientific researches with Dr. Ren Dawei and Professor Shi Yongkui in recent years. He is the Corresponding author for this academic paper. 
International Journal of Signal Processing, Image Processing and Pattern Recognition Vol.8, No.11 (2015) 